\title{
Front Matter: Volume 9823
}

, "Front Matter: Volume 9823," Proc. SPIE 9823, Detection and Sensing of Mines, Explosive Objects, and Obscured Targets XXI, 982301 (6 July 2016); doi: $10.1117 / 12.2244407$

SPIE. Event: SPIE Defense + Security, 2016, Baltimore, MD, United States 


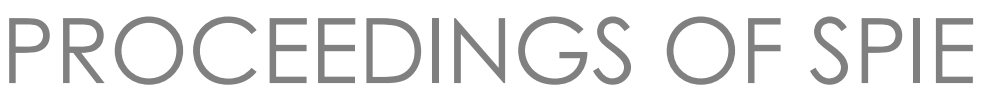

\title{
Detection and Sensing of Mines, Explosive Objects, and Obscured Targets XXI
}

\author{
Steven S. Bishop \\ Jason C. Isaacs \\ Editors
}

18-21 April 2016

Baltimore, Maryland, United States

Sponsored and Published by

SPIE 
The papers in this volume were part of the technical conference cited on the cover and title page. Papers were selected and subject to review by the editors and conference program committee. Some conference presentations may not be available for publication. Additional papers and presentation recordings may be available online in the SPIE Digital Library at SPIEDigitallibrary.org.

The papers reflect the work and thoughts of the authors and are published herein as submitted. The publisher is not responsible for the validity of the information or for any outcomes resulting from reliance thereon.

Please use the following format to cite material from this book:

Author(s), "Title of Paper," in Detection and Sensing of Mines, Explosive Objects, and Obscured Targets XXI, edited by Steven S. Bishop, Jason C. Isaacs, Proceedings of SPIE Vol. 9823 (SPIE, Bellingham, WA, 2016) Six-digit Article CID Number.

ISSN: 0277-786X

ISSN: 1996-756X (electronic)

ISBN: 9781510600645

Published by

SPIE

P.O. Box 10, Bellingham, Washington 98227-0010 USA

Telephone +1 3606763290 (Pacific Time) · Fax +1 3606471445

SPIE.org

Copyright (C) 2016, Society of Photo-Optical Instrumentation Engineers.

Copying of material in this book for internal or personal use, or for the internal or personal use of specific clients, beyond the fair use provisions granted by the U.S. Copyright Law is authorized by SPIE subject to payment of copying fees. The Transactional Reporting Service base fee for this volume is $\$ 18.00$ per article (or portion thereof), which should be paid directly to the Copyright Clearance Center (CCC), 222 Rosewood Drive, Danvers, MA 01923. Payment may also be made electronically through CCC Online at copyright.com. Other copying for republication, resale, advertising or promotion, or any form of systematic or multiple reproduction of any material in this book is prohibited except with permission in writing from the publisher. The CCC fee code is 0277-786X/16/\$18.00.

Printed in the United States of America.

Publication of record for individual papers is online in the SPIE Digital Library.

\section{SPIE. DIGITAL}

Paper Numbering: Proceedings of SPIE follow an e-First publication model, with papers published first online and then in print. Papers are published as they are submitted and meet publication criteria. A unique citation identifier (CID) number is assigned to each article at the time of the first publication. Utilization of CIDs allows articles to be fully citable as soon as they are published online, and connects the same identifier to all online, print, and electronic versions of the publication. SPIE uses a six-digit CID article numbering system in which:

- The first four digits correspond to the SPIE volume number.

- The last two digits indicate publication order within the volume using a Base 36 numbering system employing both numerals and letters. These two-number sets start with 00, 01, 02, 03, 04, $05,06,07,08,09,0 A, 0 B \ldots$ OZ, followed by 10-1Z, 20-2Z, etc.

The CID Number appears on each page of the manuscript. The complete citation is used on the first page, and an abbreviated version on subsequent pages. 


\title{
Contents
}

\author{
ix Authors \\ xi Conference Committee
}

SESSION 1 HAND-HELD SENSOR DESIGN, AND SYSTEMS TESTING

982302 Coil design considerations for a high-frequency electromagnetic induction sensing instrument [9823-1]

982303 Improved feedback amplifier for electromagnetic induction sensors [9823-2]

982304 Formulation for a practical implementation of electromagnetic induction coils optimized using stream functions [9823-3]

982305 Dynamic EMI sensor platform for digital geophysical mapping and automated clutter rejection for CONUS and OCONUS applications [9823-4]

SESSION 2 HAND-HELD EMI SENSING I

982307 Landmine detection with Bayesian cross-categorization on point-wise, contextual and spatial features [9823-6]

982308 A high power EMI sensor for detecting and classifying small and deep targets [9823-7]

982309 Adaptive coherence estimator (ACE) for explosive hazard detection using wideband electromagnetic induction (WEMI) [9823-8]

9823 OA Buried object detection using handheld WEMI with task-driven extended functions of multiple instances [9823-9]

SESSION 3 HAND-HELD EMI SENSING II

$98230 \mathrm{C}$ Computation of the eddy-current modes of three-dimensional conducting bodies [9823-11]

9823 OD Carbon fiber and void detection using high-frequency electromagnetic induction techniques [9823-12]

$9823 \mathrm{OE} \quad$ Improved electromagnetic induction processing with novel adaptive matched filter and matched subspace detection [9823-13] 
9823 OF Pulsed THz TDS of objects covered by disordered structure [9823-14]

9823 OG Evaluation of the use of 3D printing and imaging to create working replica keys [9823-15]

\section{SESSION 5 HAND-HELD GPR TECHNOLOGIES}

$9823 \mathrm{OH} \quad$ Curvelet filter based prescreener for explosive hazard detection in hand-held ground penetrating radar [9823-16]

9823 Ol Background adaptive division filtering for hand-held ground penetrating radar [9823-17]

9823 OK On the use of log-gabor features for subsurface object detection using ground penetrating radar [9823-19]

$9823 \mathrm{OL}$ Comparative analysis of short and long GPR pulses for landmine detection [9823-20]

9823 OM A label propagation approach for detecting buried objects in handheld GPR data [9823-21]

9823 ON Detecting buried explosive hazards with handheld GPR and deep learning [9823-22]

\section{SESSION 6 EM IMAGING SENSORS AND TECHNIQUES}

982300 Advanced EMI models for survey data processing: targets detection and classification [9823-23]

9823 OP Electromagnetic induction imaging of concealed metallic objects by means of resonating circuits [9823-24]

$98230 Q \quad$ Electromagnetic imaging with atomic magnetometers: a novel approach to security and surveillance [9823-25]

9823 OR Enhanced buried UXO detection via GPR/EMI data fusion [9823-26]

\section{SESSION 7 HAND-HELD SENSOR FUSION TECHNIQUES}

9823 OS Identification of improvised explosives residues using physical-chemical analytical methods under real conditions after an explosion [9823-28]

9823 OT A comparison of robust principal component analysis techniques for buried object detection in downward looking GPR sensor data [9823-29]

9823 OU Evaluation of a biomimetic optical-filter based chemical sensor for detection of hazardous chemical vapors in the infrared [9823-31] 
9823 OV Laser induced x-ray 'RADAR' particle physics model [9823-32]

9823 OY NQR detection of explosive simulants using RF atomic magnetometers [9823-35]

$98230 Z$ Polarization enhanced Nuclear Quadrupole Resonance with an atomic magnetometer [9823-36]

982311 Effectiveness of laser sources for contactless sampling of explosives [9823-38]

982312 Digital micromirror devices in Raman trace detection of explosives [9823-39]

\section{SESSION 9 ROAD DETECTION AND SCENE MODELING}

982313 Improving the detection of explosive hazards with LIDAR-based ground plane estimation [9823-40]

982314 3D environment modeling and location tracking using off-the-shelf components [9823-41]

982315 Road detection in arid environments using uniformly distributed random based features [9823-42]

\section{SESSION 10 OVERHEAD SENSING}

982316 Integrated use of field spectroscopy and satellite remote sensing for defence and security applications in Cyprus (Invited Paper) [9823-43]

982317 Roadside IED detection using subsurface imaging radar and rotary UAV [9823-44]

982318 Integration of micro-fabricated atomic magnetometers on military systems [9823-45]

\section{SESSION 11 DOWN-LOOKING GPR TECHNIQUES}

982319 Attribute-driven transfer learning for detecting novel buried threats with groundpenetrating radar [9823-46]

$98231 \mathrm{~A}$ Algorithm development for deeply buried threat detection in GPR data [9823-47]

9823 IB Enhancements to GPR buried UXO detection using the apex-shifted hyperbolic radon tansform [9823-48]

9823 1C A fisher vector representation of GPR data for detecting buried objects [9823-49]

9823 ID Fusion of KLMS and blob based pre-screener for buried landmine detection using ground penetrating radar [9823-50]

$98231 \mathrm{E} \quad$ Preprocessing of A-scan GPR data based on energy features [9823-51] 


\section{SESSION 12 FORWARD LOOKING LWIR FUSION, EVALUATION LWIR AND MWIR, AND LDV SEISMIC}

PROCESSING

9823 IF Anomaly detection using classified eigenblocks in GPR image [9823-52]

$98231 \mathrm{G} \quad$ Multiple kernel based feature and decision level fusion of iECO individuals for explosive hazard detection in FLIR imagery [9823-53]

\section{SESSION $13 \quad$ FORWARD LOOKING GPR TECHNIQUES}

$982311 \quad$ A feature learning approach for classifying buried threats in forward looking ground penetrating radar data [9823-56]

$98231 \mathrm{~J} \quad$ Convolutional neural network based sensor fusion for forward looking ground penetrating radar [9823-57]

$98231 \mathrm{~K} \quad$ Using queuing models to aid design and guide research effort for multimodality buried target detection systems [9823-58]

$98231 \mathrm{~L} \quad$ Sequential feature selection for detecting buried objects using forward looking ground penetrating radar [9823-60]

$98231 \mathrm{M}$ Spectral diversity for ground clutter mitigation in forward-looking GPR [9823-61]

\section{SESSION 14 SIDE-SCANNING SENSING, DATA PROCESSING, AND PROGRAMS I}

9823 iN Multiple instance learning for buried hazard detection [9823-62]

982310 Multiple-modality program for standoff detection of roadside hazards [9823-63]

9823 IP Advances in ground vehicle-based LADAR for standoff detection of road-side hazards [9823-64]

$98231 Q \quad$ Explosive hazard detection using synthetic aperture acoustic sensing [9823-65]

9823 IR Comparison of spatial frequency domain features for the detection of side attack explosive ballistics in synthetic aperture acoustics [9823-66]

9823 is Detection of landmines and UXO using advanced synthetic aperture radar technology [9823-67]

SESSION 15 SIDE-SCANNING SENSING, DATA PROCESSING, AND PROGRAMS II

9823 1T Statistically normalized coherent change detection for synthetic aperture sonar imagery [9823-68]

$98231 \mathrm{U}$ Optimized passive sonar placement to allow improved interdiction [9823-69] 
$98231 \mathrm{~V} \quad$ Risk-based scheduling of multiple search passes for UUVs [9823-70]

9823 IW Edge defection of red hind grouper vocalizations in the littorals [9823-71]

$98231 \mathrm{X} \quad$ Multi-input multi-output waveform optimization for synthetic aperture sonar [9823-72] 
Proc. of SPIE Vol. $9823982301-8$

Downloaded From: https://www.spiedigitallibrary.org/conference-proceedings-of-spie on 25 Apr 2023 Terms of Use: https://www.spiedigitallibrary.org/terms-of-use 


\section{Authors}

Numbers in the index correspond to the last two digits of the six-digit citation identifier (CID) article numbering system used in Proceedings of SPIE. The first four digits reflect the volume number. Base 36 numbering is employed for the last two digits and indicates the order of articles within the volume. Numbers start with 00, 01, 02, 03, 04, 05, 06, 07, 08, 09, OA, OB...0Z, followed by 10-12, 20-2Z, etc.

Agapiou, Athos, 16

Aggarwal, Ishwar D., OU

Ågren, Matilda, 12

Akar, Gözde Bozdaği, 1D

Akmalov, Artem E., 11

Aksoy, Serkan, OL

Alexson, Dimitri A., OY, $\mathrm{OZ}$

Alvey, Brendan, 09

Anderson, Derek T., OH, OI, 1G, IR

Ball, John E., OH, OI, IR

Barrall, Geoffrey A., $\mathrm{OZ}$

Barrowes, Benjamin E., 02, 08, 0D, 00

Bartlett, P. A., OP

Baydar, Bora, 1D

Baylog, John G., IV

Beaujean, Pierre-Philippe, $1 \mathrm{~W}$

Bennett, Hollis J., 02, OD

Beroun, Ivo, OS

Besaw, Lance E., ON

Bischeltsrieder, Florian, $1 S$

Brewster, E., 1Q

Buck, A., 13

Burns, Brian P., 1L

Burns, Joseph, OR, OT

Camilo, Joseph A., 11

Chen, David, $1 \mathrm{~J}$

Chistyakov, Alexander A., 11

Close, Ryan, 10, 1P

Collins, Leslie M., 19, 1A, 11, 1K

Colwell, Kenneth A., 19

Cook, Matthew, 09, 0A

Crosskey, Miles, $1 \mathrm{~J}$

Deans, Cameron, $0 Q$

Deas, R., OV

Deguzman, P. C., OU

Dill, Stephan, 15

Dogan, Mesut, $1 \mathrm{E}$

Dowdy, Josh, IR

Dunnill, Kevin F., OU

Espy, Michelle A., OZ

Ewing, Kenneth J., OU

Frigui, Hichem, OM, 1C

Gabbay, Jonathan E., OC

Gallivan, Kyle A., IX

Gandhe, Avinash, 07

Glimtoft, Martin, 12

G-Michael, Tesfaye, $1 \mathrm{~T}$

Guilizzoni, R., OP

Hadjimitsis, Diofantos G., 16
Harris, Samuel, OK

Havens, Timothy C., OR, OT, 1G, 1M, 1N, 1R

Hayes, Charles Ethan, OE

Heinzel, Andreas, 15

Ho, Dominic K. C., 09, 0A, 0K, $1 \mathrm{~L}$

Hollinger, Jim, IP

Hu, Lequn, $1 \mathrm{G}$

Huang, Wen, $1 X$

Hussain, Sarah, $0 Q$

Johnson, Bruce A., $1 \mathrm{U}$

Karem, Andrew, 1C

Keller, James M., 13, 15, 1G, 1L, 1Q, 1R

Kelly, Jack, OR

Keranen, Joe, 05

Kerlin, Scott, OG

Kerr, Andrew J., OE

Khalifa, Amine B., 1C

Kim, Min Ju, $1 F$

Kim, Seong Dae, IF

Kotkovskii, Gennadii E., 11

Kotrlý, Marek, OS

Lai, C. P., 17

Laudato, Stephen J., 05

Le, Viet Q., 17

Lee, Matthew A., 0 l

Lee, Seung-eui, $1 \mathrm{~F}$

Léveillé, Jasmin, 07

Lockley, D., OV

Luke III, Robert H., 14, 1G, 1L, 10, 1R

Major, Kevin J., OU

Malof, Jordan M., 1A, 11, 1K

Malone, Michael W., OZ

Marchand, Bradley, IX

Marchand, Melissa, 1X

Mareš, Bohumil, OS

Marmugi, Luca, $0 Q$

Masarik, Matthew P., OR, OT, IB

Matthews, Cameron A., IU, IW

McClellan, James H., OE

Melillos, George, 16

Mhaskar, Rahul, 18

Michaelides, Silas, 16

Middleton, Seth, 10, 1P

Miller, Jonathan S., 05, 18

Monti, Mark C., OY, OZ

Morton, Kenneth, 1J

Moss, R., OV

Murray, Bryce, $1 \mathrm{G}$

Nazlı, Hakkı, OL 
Neely, D., OV

Nguyen, Son, 1P

Nordberg, Markus, 12

Okamitsu, Jeffrey K., OY, OZ

O'Neill, Kevin A., 02, 08, 0D, 00

Östmark, Henric, 12

Öztürk, Serhat, 1D

Papadavid, George, 16

Parker, Brian, $\mathrm{OH}$

Peichl, Markus, is

Pinar, Anthony, OT, IN

Plodpradista, P., 15

Popescu, M., 13, 15, 1Q

Poutous, Menelaos K., OU

Price, Stanton R., $1 \mathrm{G}$

Prodromou, Maria, 16

Prouty, Mark, 18

Qin, Yexian, 17

Reed, Mark A., 04

Reichman, Daniël, 1A

Reid, Graham, OM

Ren, Yu-Jiun, 17

Renzoni, Ferruccio, OP, OQ

Rice, Joseph, OT, IN

Roberts, Rodney G., $1 \mathrm{~T}$

Rupp, Ronald, $1 \mathrm{P}$

Rusby, D., OV

Sakaguchi, Rayn, $1 \mathrm{~J}$

Sanghera, Jasbinder S., OU

Schreiber, Eric, 1S

Schultz, Gregory, 05, 18

Schulz, Timothy J., 1M, 1N

Scott, Waymond R., 03, 04, OC, OE

Shamatava, Irma, 08, 00

Shaw, Darren, $1 \mathrm{~L}$

Shubitidze, Fridon, 02, 08, 0D, 00

Sigman, John Brevard, 02, 08, 0D, 00

Simms, Janet E., 02, OD

Steinhurst, Daniel A., 08

Stone, Kevin, $1 \mathrm{~L}, 1 \mathrm{Q}$

Straub, Jeremy, $0 G$

Suri, Rajiv, 10

Svanqvist, Mattias, 12

Temlioğlu, Eyyup, OL

Thelen, Brian T., OR, OT, 1B

Themistocleous, Kyriacos, 16

Trofimov, V. A., OF

Tucker, J. Derek, IT

Turhan-Sayan, Gonul, $1 \mathrm{E}$

Turková, Ivana, OS

Twumasi, Jones O., 17

Varentsova, Svetlana A., OF

Vessey, Alyssa, 1P

Walenz, Brett, $1 \mathrm{~J}$

Wang, Yinlin, 02, 08, 0D, 00

Watson, J. C., OP

Webb, Adam, 1M, 1N

Wettergren, Thomas A., IV

White, Julie L., $\mathrm{OH}, \mathrm{OI}$

Williams, Kathryn, 10, 1P
Wilson, L. A., OV

Xique, Ismael J., 1B

Yu, Ssu-Hsin, 07

Yu, Tzuyang, 17

Yüksel, Seniha Esen, 1D

Yule, Donald E., 02, OD

Zagursky, D. YU., OF

Zakharova, I. G., OF

Zare, Alina, 09, 0A, OK 


\title{
Conference Committee
}

\author{
Symposium Chair
}

David A. Logan, BAE Systems (United States)

Symposium Co-chair

Donald A. Reago Jr., U.S. Army Night Vision \& Electronic Sensors

Directorate (United States)

Conference Chairs

Steven S. Bishop, U.S. Army Night Vision \& Electronic Sensors

Directorate (United States)

Jason C. Isaacs, Naval Surface Warfare Center Panama City Division (United States)

Conference Program Committee

Benjamin E. Barrowes, U.S. Army Engineer Research and Development Center (United States)

Ryan R. Close, U.S. Army Night Vision \& Electronics Sensors Directorate (United States)

Leslie M. Collins, Duke University (United States)

Gerald J. Dobeck, Naval Surface Warfare Center Panama City Division (United States)

Anthony A. Faust, Defence Research and Development Canada, Suffield (Canada)

Tesfaye G-Michael, Naval Surface Warfare Center Panama City Division (United States)

Gregory Garcia, Naval Surface Warfare Center Panama City Division (United States)

James M. Keller, University of Missouri-Columbia (United States)

Aaron LaPointe, U.S. Army Night Vision \& Electronic Sensors

Directorate (United States)

Henric Östmark, Swedish Defence Research Agency (Sweden)

Motoyuki Sato, Tohoku University (Japan)

Waymond R. Scott Jr., Georgia Institute of Technology (United States)

Richard C. Weaver, U.S. Army Night Vision \& Electronic Sensors

Directorate (United States) 
Session Chairs

1 Hand-Held Sensor Design, and Systems Testing

Ken E. Yasuda, U.S. Army Night Vision \& Electronic Sensors

Directorate (United States)

Lance E. Besaw, Applied Research Associates, Inc. (United States)

2 Hand-Held EMI Sensing I

Ken E. Yasuda, U.S. Army Night Vision \& Electronic Sensors

Directorate (United States)

Joe Keranen, White River Technologies, Inc. (United States)

3 Hand-Held EMI Sensing II

Brian C. Barlow, U.S. Army Night Vision \& Electronic Sensors

Directorate (United States)

Gregory Schultz, White River Technologies, Inc. (United States)

4 THz Time Domain Spectroscopy of Objects, and 3D Contraband

Scanning

Brian C. Barlow, U.S. Army Night Vision \& Electronic Sensors

Directorate (United States)

Dominic K. Ho, University of Missouri (United States)

$5 \quad$ Hand-held GPR Technologies

Waymond R. Scott Jr., Georgia Institute of Technology (United States)

Rajiv Suri, U.S. Army RDECOM CERDEC NVESD (United States)

6 EM Imaging Sensors and Techniques

James C. Shpil, U.S. Army Night Vision \& Electronic Sensors

Directorate (United States)

Alina Zare, University of Missouri (United States)

7 Hand-held Sensor Fusion Techniques

Ken E. Yasuda, U.S. Army Night Vision \& Electronic Sensors

Directorate (United States)

Lance E. Besaw, Applied Research Associates, Inc. (United States)

8 Chemical Detection: Joint Session with conferences 9823 and 9824

Vincent P. Schnee, U.S. Army Night Vision \& Electronic Sensors

Directorate (United States)

Anthony A. Faust, Defence Research and Development Canada,

Suffield (Canada) 
9 Road Detection and Scene Modeling

Mihail Popescu, University of Missouri (United States)

Christopher Marshall, U.S. Army Night Vision \& Electronic Sensors

Directorate (United States)

10 Overhead Sensing

Seth Middleton, U.S. Army Night Vision \& Electronic Sensors

Directorate (United States)

Mark W. Hibbard, CoVar Applied Technologies, Inc. (United States)

11 Down-looking GPR Techniques

Leslie M. Collins, Duke University (United States)

Kathryn A. Williams, U.S. Army Night Vision \& Electronic Sensors

Directorate (United States)

12 Forward Looking LWIR Fusion, Evaluation LWIR and MWIR, and LDV Seismic Processing

Kenneth D. Morton Jr., CoVar Research (United States)

Ryan R. Close, U.S. Army RDECOM CERDEC NVESD (United States)

13 Forward Looking GPR Techniques

Miles Crosskey, CoVar Applied Technologies, Inc. (United States)

Brian P. Burns, U.S. Army RDECOM CERDEC NVESD (United States)

14 Side-scanning Sensing, Data Processing, and Programs I

Robert H. Luke III, U.S. Army Night Vision \& Electronic Sensors

Directorate (United States)

Timothy C. Havens, Michigan Technological University (United States)

15 Side-scanning Sensing, Data Processing, and Programs II

Julia Gazagnaire, Naval Surface Warfare Center Panama City

Division (United States)

Tesfaye G-Michael, Naval Surface Warfare Center Panama City

Division (United States) 
Proc. of SPIE Vol. $9823982301-14$

Downloaded From: https://www.spiedigitallibrary.org/conference-proceedings-of-spie on 25 Apr 2023 Terms of Use: https://www.spiedigitallibrary.org/terms-of-use 